\title{
The Role of Diagnostic Tissue in Research
}

\author{
Carol C. Cheung ${ }^{a, c}$ Emina E. Torlakovic ${ }^{b, c}$ Anna Porwit ${ }^{b, c}$ \\ Departments of a Pathology and ${ }^{\mathrm{b}}$ Laboratory Hematology, University Health Network, and ${ }^{\mathrm{C}}$ Department of \\ Laboratory Medicine and Pathobiology, Faculty of Medicine, University of Toronto, Toronto, Ont., Canada
}

\author{
Key Words \\ Diagnostic tissue - Pathology - Access to diagnostic tissue · \\ Research · Biobanking · Residual tissue
}

\begin{abstract}
There are two broad classes (or categories) of excised human tissue: diagnostic tissue (DT) and research tissue (RT). Classification of excised human tissue does not define its ultimate use and ultimate use of excised human tissue does not define its classification. While both DT and RT can be used for research, DT has specific requirements with respect to how it must be handled if and when being accessed for research. We highlight distinguishing features of DT: (1) it is a clinical record, (2) it must be identifiable to a specific individual, (3) it is stewarded by pathology departments/clinical laboratories and (4) it has a mandatory retention period. We discuss how the further sub-classification of DT into archived DT (aDT) and excess DT (eDT) impacts the nature of its role in research. We examine the concept of DT as a clinical record and emphasize the impact of mandatory retention as it applies to how DT may be accessed for research purposes. We explain the role of post-retention eDT as a source of RT as well as procedures for access to in-retention aDT for research. Clarity of such issues will facilitate responsible access to DT for research.

(c) 2015 S. Karger AG, Basel
\end{abstract}

\section{Introduction}

Diagnostic tissue (DT) is defined as all tissue removed for a medically indicated procedure that in accordance with applicable regulatory requirements must be sent to the clinical laboratory for analysis, reporting, retention and ultimately, disposal in an appropriately safe manner [1]. In addition to its primary use for patient care, as the largest available source of clinically (including pathologically) annotated excised human tissue [2], DT has always been, and will continue to be, secondarily used for many types of research including basic science research, translational research and prospective clinical trials [3-6]. The importance of DT for research, especially for retrospective studies, will only increase as personalized medicine continues to evolve. The need to clearly examine the role of DT in research has been brought about by two major trends: (1) the demand for human tissue in the era of personalized medicine is increasing and (2) the emphasis on early detection and 'minimally invasive' techniques have resulted in smaller specimens that has an impact on the amount of DT available for both primary use (i.e. patient care) and secondary use (e.g. future patient care, quality assurance, medicolegal investigations, education and, of course, research). As DT is a part of an individual patient's clinical record, access is administered by its institutional stewards, which are typically the pathology departments/clinical laboratories [1,7]. The principles and practicalities of managing access to DT from the stewardship perspective of a clinical department of pathology for

Carol C. Cheung

Department of Pathology, University Health Network

200 Elizabeth Street, 11th Floor, Eaton Wing

Toronto, ON M5G 2C4 (Canada)

E-Mail c.cheung@utoronto.ca 
all types of requests have been articulated elsewhere [4]. This article specifically addresses the two main roles for DT in research. The first or 'indirect' role is that in certain circumstances DT may be a source of research tissue (RT). The second or 'direct' role is that DT may itself be directly accessed for research experiments. To do this, we will discuss the classification of tissue versus the use of tissue, distinguishing features of DT relevant to research, postretention DT as a source of RT, direct access to in-retention DT for research and conclude by emphasizing the importance of these concepts in facilitating access to DT for research. Where appropriate, we will refer to processes at University Health Network (UHN), Toronto, Ont., which is a large quaternary care academic hospital in Canada, where requests for access to DT for all purposes (including but not limited to research) are overseen by the Access to Diagnostic Tissue Committee (ADTC) [4].

\section{Classification of Tissue versus Use of Tissue}

In the simplest terms, DT is all tissue removed for a medically indicated procedure, while RT is all tissue removed for a research-only procedure. This initial dichotomous classification, which is based on the original reason for removal and can be distinguished using the modified 'but for' construct [1], is important because DT must follow specific rules with respect to handling, retention and disposal. There are two main misconceptions relating to the classification and use of excised human tissue: (1) that classification defines use and (2) that use defines classification. The first misconception stems from the erroneous belief that only RT can be used for research and that because of this, excised human tissue should not be classified as either DT or RT, but should remain 'both' [8]. This is neither true nor necessary because classification of tissue does not define use. Both RT and DT not only may be, but in practice are, used for research - it is simply that additional considerations must be taken into account when it comes to using DT for research, which we will further discuss below. This then leads to the second misconception, which stems from the erroneous belief held by some that if researchers want to use tissue for research, then that tissue should be considered RT regardless of its true classification and that the rules for access should be those for RT rather than for DT. This is not true because use does not define classification. Therefore, while both RT and DT may be accessed for research, it is important to recognize that specific considerations must be taken into account when such access involves DT.

\section{Distinguishing Features of DT Relevant to Research}

There are four important distinguishing features related to the potential use of DT for research: (1) it is a clinical record of the institution/clinical laboratory, (2) it must be identifiable to a specific individual, (3) it is under the stewardship of the clinical laboratory and (4) it has a mandatory retention period. These four features are all important in determining the nature of the role of DT in research.

\section{DT as a Clinical Record}

$\mathrm{DT}$, in all its forms, is primarily a clinical record of the care provided by the institution/clinical laboratory to a patient; in contrast, RT is primarily a resource specifically set aside for research. This represents a source of confusion in situations where researchers who want to use DT for research, especially clinical paraffin tissue blocks, sometimes argue that DT is not being retained as a record, but rather as a resource for research. Proponents of this argument often claim that unless each type of DT is explicitly stated in law as being part of a record, then it should not be considered as such (i.e. the 'show me exactly where in the law it specifically says that' argument). While it is beyond the scope of this article to perform an international comparative analysis of approaches to statutory interpretation, unless there is specific language that states otherwise, then in general, 'absence of proof is not necessarily proof of absence'. On more practical terms, however, we will simply note that the argument is of limited relevance in the real world. Clinical laboratories have specific policies and procedures to retain the different kinds of DT (e.g. wet tissue, glass slides, paraffin blocks, extracted derivatives, etc.). If DT were being retained as a resource for research, then presumably this would only be possible for clinical laboratories that specifically receive research funding to retain DT for research and that the terms of the retention would be under the oversight of a local research ethics board (REB); however, neither of these situations are the norm in the real world. In addition, clinical laboratories that are 'non-academic' and 'purely clinical' also retain DT in accordance with specific policies and procedures similar to those of academic centres. Therefore, if not being retained specifically for research, then any request to access DT for research becomes one of secondary use.

In the end, clinical laboratories themselves view DT as a record of the clinical care provided to a patient and retain them as such $[4,5,7,9]$. DT may be accessed for review or follow-up testing that may be required when 
diagnostic specimens are sent for second opinion, consultation, medicolegal investigation, for quality assurance to ensure that any testing being performed by our laboratories are properly controlled, for education, and for research. Therefore, while DT may certainly be legitimately accessed for research purposes, this is not the sole reason for which clinical laboratories retain DT.

\section{Identifiability of DT}

DT must be identifiable to a specific individual, just like other clinical records. In contrast, RT is de-identified and usually coded (i.e. where '...direct identifiers are removed from the materials and replaced with a code. Depending on access to the code, it may be possible to reidentify specific individuals.' [10]), which is typically required by REBs [10,11]. Therefore, since all DT is identifiable, if and when released for research, issues of privacy and de-identification need to be given the highest consideration.

\section{Stewardship of DT}

Clinical laboratories or pathology departments are the stewards of DT; RT on the other hand does not have to be stewarded by the clinical laboratory, but may instead be stewarded by a research biobank. It is worth noting that stewardship is distinct from ownership [4, 7]. True ownership of human tissue is a complex topic $[1,4,7,12-15]$ and may vary internationally depending on how specifically its legal status has been established by different countries. While it is beyond the scope of this article to engage in such debate or to perform a comparative analysis of international approaches to tissue ownership, we will say this: stewards are not owners and that issues of ownership are irrelevant to the practical role of the clinical laboratory as stewards of DT [4]. DT is a record and it is the responsibility of its steward to ensure that the integrity of the record is protected.

\section{Mandatory Retention of DT}

The lifecycle of DT consists of four mandatory phases: acquisition, evaluation/analysis, retention and disposal [4]. It is a common misconception that once a pathology report has been issued, which occurs at the end of the evaluation/analysis phase, DT is considered 'left over' and 'no longer needed'. This is not true because post-analysis DT represents a record of the care provided by the institution (or laboratory); as such, retention of DT is mandatory. DT is only legitimately considered as 'left over' once the mandatory retention period has elapsed just prior to disposal. Two factors relating to the retention phase are relevant for research: the sub-classification of DT and the retention status of DT.

Sub-Classification of DT Impacts on the Relative Length of Its Retention Period. After excision, DT that undergoes subsequent sampling and processing becomes archived DT (aDT); this includes not only the formalin-fixed paraffin-embedded tissue blocks and glass slides that are handled by clinical laboratories on a daily basis, but also frozen materials and any extracted tissue derivatives. DT that does not undergo subsequent sampling and processing becomes excess DT (eDT); this is typically what remains as wet, formalin-fixed tissue after a specimen has been grossed and blocked. The subclassification of DT is important because it determines the relative length of the retention period; $\mathrm{aDT}$ is retained long term (i.e. years), while $\mathrm{eDT}$ is retained short term (i.e. weeks).

Retention Status ofDT Impacts on Its Role in Research. DT that is still within its mandatory retention period is in-retention DT; in-retention DT is kept in the clinical archives of the institution's clinical laboratories. After the mandatory retention period of in-retention DT has elapsed, it becomes post-retention DT. Post-retention DT has 3 potential fates: (1) it may be incinerated as medical waste, (2) it may remain in the clinical archives of the clinical laboratory or (3) it may be transferred to a research biobank if appropriate conditions are met. Therefore, post-retention DT may become a source of RT, which in turn may be accessed for research experiments from the stewards of RT (e.g. research biobanks).

\section{Post-Retention DT as a Source of RT}

While RT can be obtained directly via research-only procedures, it can also be obtained indirectly from postretention DT. This process requires approval from (1) the current stewards (i.e. the clinical laboratory) to officially release the post-retention tissue, (2) the future stewards (e.g. the research biobank) to officially accept the tissue and (3) the REB to provide oversight for considerations around specific research consent for banking of tissue for future research and proper de-identification/coding of samples $[6,16,17]$. After these prerequisites are fulfilled, the post-retention DT becomes RT and there is an official transfer of stewardship from the clinical laboratory to the research biobank. Subsequent requests to use this material for specific research experiments will then follow the process for access to RT including specific consent from a research participant or 
REB waiver of consent and approval from the stewards of the RT (e.g. the research biobank). Both post-retention $\mathrm{EDT}$ and post-retention $\mathrm{aDT}$ may be a source of RT. However, due to its relatively short mandatory retention period, post-retention $\mathrm{eDT}$ is the most common indirect source of RT.

\section{Post-Retention eDT as a Source of RT}

In this scenario, after an excised specimen has been sampled for further processing and analysis, what remains as eDT (i.e. formalin-fixed wet tissue), rather than being incinerated after its mandatory retention period, may be banked as RT [18]. Depending on institutional policies and procedures, the physical act of banking eDT may actually take place while it is still within its mandatory retention period; however, from an administrative perspective, it may not be officially released by the clinical laboratory until the retention period has elapsed. This allows material that, based on the experience and expertise of the clinical laboratory, would otherwise be incinerated, to be obtained and processed at a time that is more optimal for preservation of possible future analyses of in situ and extracted tissue components (e.g. immunohistochemistry, FISH, mutational analyses, gene expression signatures, etc.). Such a practice would also allow for fresh-frozen tissue to be banked for research in addition to the usual formalin-fixed paraffin-embedded tissue [18]. Because stewardship is not officially transferred until the mandatory retention period is over, it allows the clinical laboratory the opportunity to 'recall' the 'anticipatorily banked' eDT in the rare event that it may end up being required for the clinical analysis necessary to generate the initial pathology medical report.

This flexibility is made possible by the short retention period of the eDT and works best where the clinical laboratory and the research biobank work closely together, ideally within the same institution [18]. This is just the case at UHN, where the research banking of $\mathrm{eDT}$ is a collegial and collaborative effort between the Department of Pathology of the Laboratory Medicine Program (LMP) that operates the UHN clinical laboratories and the REB-sanctioned Biospecimen Sciences Program that operates the UHN research biobank.

\section{Post-Retention aDT as a Source of RT}

Post-retention aDT may also be a source of RT. Two major differences exist from the post-retention eDT route. The first is the longer mandatory retention period for $\mathrm{aDT}$. The second is that privacy considerations are more complex since aDT already exists in forms prede- termined by the needs of clinical care and by definition is already labeled with patient identifiers. For example, paraffin blocks that may have already been engraved with patient identifiers would need to undergo an additional step of removing the patient identifiers prior to proper coding of the samples.

\section{Direct Access to In-Retention DT for Research}

Access to DT is administered by its designated stewards, which are usually institutional pathology departments or the entity that operates the clinical laboratories. The specific nuances of this process may differ from one country to another, or from one institution to another, but it typically begins with a request from a principal investigator or his/her delegate such as a research coordinator. There is consideration of the request by the head of the clinical lab or his/her delegate, which may be either a person or an access to DT committee. Access to DT is approved for those requests that have specific testing for the requested tissue, after which the tissue is released to the researcher if (1) it is present in sufficient amounts, (2) fulfilling the request will not alter any histologic findings and (3) there is specific participant consent and/or REB-approved waiver of specific participant consent. The clinical laboratory is typically compensated for the administrative, technical and professional components of reviewing and processing the request. From the above, we see that there are two phases involved in the request: an approval phase and a release phase.

At UHN, it is the ADTC of the LMP that oversees requests for access to and ultimately release of DT from an administrative perspective for all purposes including research; the principles and practicalities of the UHNLMP-ADTC approach to DT have been previously published [4]. Briefly, for research requests involving central review, digital slides are approved. For research requests involving additional testing using DT (mainly aDT that has been formalin fixed and paraffin embedded), ADTC will approve access to unstained slides in an amount sufficient for the specific testing in the study/trial protocol. Once approved, a two-step suitability and adequacy assessment is performed after which the tissue is released. ADTC reviews occur primarily from an administrative perspective of stewards who are responsible for preserving the integrity of DT as a clinical record. The responsibilities for primary scientific and ethical review are performed by other bodies (e.g. REB) and are not part of 
the direct duties of the ADTC. All research requests are referred to the appropriate bodies within the LMP for cost recovery. From a practical perspective, the ADTC considers research as anything that must follow a study/ trial protocol, requires REB approval and/or requires participants to sign a research consent form for participation in a research study/trial.

A special type of request involving access to in-retention DT that may arise in the research context is that of external banking. External banking is the phenomenon where an external researcher or research organization requests that tissue be physically transferred to them for currently unspecified, possible future research [4]. External banking requests involving RT may be a simple question of resource allocation for the stewards of RT to consider. External banking requests involving DT, however, are different in nature because unlike RT, DT is not primarily a resource. Requests to externally bank in-retention DT involve the transfer of original clinical records, or a portion thereof, that are still within their mandatory retention periods to an external research repository for possible use in possible future research. At UHN, the ADTC experience has been that while external banking requests may stand alone, more often they manifest as a request where some tissue is requested for specific testing and some tissue is requested for possible future use (i.e. banking). Alternatively, this type of request may also manifest as one where there is specific tissue testing described in the study protocol, but the request includes a stipulation for an amount of tissue that exceeds what is reasonably necessary for the described testing; the tissue that would be 'leftover' from such a request would presumably be banked for possible future research. Typically, the ADTC will approve access to DT for the portion of the request that involves specific testing in an amount sufficient to perform the described testing, but the ADTC will not approve the portion of the request that involves external banking of DT for possible future use. If and when additional specific testing is actually to be performed at a future date, a request for additional material with an amended or companion protocol can be submitted at that time.

Research requests for access to post-retention DT are also typically handled by the clinical laboratory. This is because post-retention DT is still a record, still identifiable to a specific individual and still under the stewardship of the clinical laboratory. It represents clinical records that the clinical laboratory may choose to keep, but does not have to. In this way, while the clinical laboratory has more options with what may be done with postretention DT (see 'Sub-Classification of DT Impacts on the Relative Length of Its Retention Period'), if it is to remain in the clinical archives, it should be treated with the same level of responsibility as in-retention DT.

\section{Conclusion}

While specifics may vary between different countries and institutions, the concepts we have presented here are widely applicable to any setting where access to DT may be requested for research. Clinical pathology departments, which typically operate institutional clinical laboratories and are therefore stewards of DT, play a central role in providing access to excised human tissue for research purposes. Clarity regarding these concepts is essential for pathology departments because it will improve accountability and thus facilitate the responsible access to excised human materials for research.
References
1 Cheung CC, Martin BR, Asa SL: Defining diagnostic tissue in the era of personalized medicine. CMAJ 2013;185:135-139.

-2 Bevilacqua G, Bosman F, Dassesse T, Hofler $\mathrm{H}$, Janin A, Langer R, Larsimont D, Morente MM, Riegman P, Schirmacher P, Stanta G, Zatloukal K, Caboux E, Hainaut P: The role of the pathologist in tissue banking: European consensus expert group report. Virchows Arch 2010;456:449-454.

3 Bathe OF, McGuire AL: The ethical use of existing samples for genome research. Genet Med 2009;11:712-715.
-4 Cheung CC: Issues with diagnostic tissue: a practical approach from a Canadian perspective. Diagn Histopathol 2013;19:337-342.

5 Giannini C, Oelkers MM, Edwards WD, Aubry MC, Muncil MM, Mohamud KH, Sandleback SG, Nowak JM, Bridgeman A, Brown ME, Cheville JC: Maintaining clinical tissue archives and supporting human research: challenges and solutions. Arch Pathol Lab Med 2011;135:347-353.

6 Riegman PH, van Veen EB: Biobanking residual tissues. Hum Genet 2011;130:357-368. 
7 Dry S, Grody WW, Papagni P: Stuck between a scalpel and a rock, or molecular pathology and legal-ethical issues in use of tissues for clinical care and research: what must a pathologist know? Am J Clin Pathol 2012;137:346355.

8 Ghent H, Petrie J: Whose tissue is it anyway? CMAJ June 10, 2013 (published online; http:// www.cmaj.ca/content/185/2/135.figures-only/reply\#cmaj_el_716136).

$\checkmark 9$ Epstein JI: Pathologists and the judicial process: how to avoid it. Am J Surg Pathol 2001; 25:527-537.

10 Tri-Council Policy Statement: Ethical Conduct for Research Involving Humans. Ottawa, Canadian Institutes of Health Research, Natural Sciences and Engineering Research Council of Canada, Social Sciences and $\mathrm{Hu}$ manities Research Council of Canada, 2010.
11 Elger BS, Caplan AL: Consent and anonymization in research involving biobanks: differing terms and norms present serious barriers to an international framework. EMBO Rep 2006;7:661-666.

12 Allen MJ, Powers ML, Gronowski KS, Gronowski AM: Human tissue ownership and use in research: what laboratorians and researchers should know. Clin Chem 2010;56: 1675-1682.

13 Dry S: Who owns diagnostic tissue blocks. Lab Med 2009;40:69-73.

14 Hakimian R, Korn D: Ownership and use of tissue specimens for research. JAMA 2004; 292:2500-2505

15 Sandor J, Bard P, Tamburrini C, Tannsjo T: The case of biobank with the law: between a legal and scientific fiction. J Med Ethics 2012; 38:347-350.
6 Gefenas E, Dranseika V, Serepkaite J, Cekanauskaite A, Caenazzo L, Gordijn B, Pegoraro R, Yuko E: Turning residual human biological materials into research collections: playing with consent. J Med Ethics 2012;38: 351-355.

17 Giesbertz NA, Bredenoord AL, van Delden JJ: Inclusion of residual tissue in biobanks: optin or opt-out? PLoS Biol 2012;10:e1001373.

18 McDonald SA, Chernock RD, Leach TA, Kahn AA, Yip JH, Rossi J, Pfeifer JD: Procurement of human tissues for research banking in the surgical pathology laboratory: prioritization practices at Washington University Medical Center. Biopreserv Biobank 2011;9: 245-251. 